\title{
Propiedades psicométricas del cuestionario multidimensional de memoria en población chilena adulta
}

\author{
Psychometric properties of multifactorial memory questionnaire in chilean adult \\ population
}

\author{
Bernardo Riffo \\ Departamento de Español, Universidad de Concepción, Chile \\ Fernando Reyes \\ Departamento de Psicología, Universidad de Concepción, Chile \\ Facultad de Psicología, Universidad del Desarrollo, Chile \\ Mónica Véliz de Vos \\ Departamento de Español, Universidad de Concepción, Chile
}

(Rec: 13 de marzo de 2012 / Acept: 03 de mayo de 2013)

\begin{abstract}
Resumen
El estudio presenta los datos de confiabilidad, validez convergente, de contenido y validez factorial del Multifactorial Memory Questionnaire [MMQ] en una muestra de 740 participantes correspondientes a población chilena normal, sin problemas de salud, en rangos de edad de 20 a 81 años. El MMQ posee 56 ítems tipo likert, correspondientes a tres dimensiones referidas a confianza en la memoria, capacidad y estrategias o ayudas usadas diariamente para recordar información. A partir del análisis realizado es posible afirmar que el instrumento presenta validez de contenido, validez factorial y confiabilidad de consistencia interna, similar a la reportada en su versión original. Se presenta además, la relación existente con sexo, edad, satisfacción vital y salud subjetiva. El MMQ es un instrumento adecuado para ser usado tanto en contextos clínicos como de investigación para evaluar metamemoria.

Palabras clave: Validez, confiabilidad, metamemoria, población adulta.
\end{abstract}

\begin{abstract}
The current study presents data on the reliability, convergent, content and factorial validity of the Multifactorial Memory Questionnaire [MMQ] in a Chilean sample of 740 normal and healthy participants aged 20 to 81 years. The MMQ is a 56-item likert scale questionnaire divided into three dimensions: memory contentment, ability, and everyday strategies or aids used to remember information. The analysis indicates that the instrument has content validity, factorial validity and internal reliability, similar to the data reported on the original study of the instrument. Data of the relationship with sex, age, life satisfaction and subjective health are also presented. The MMQ is a useful scale to evaluate metamemory in clinical and research settings. Key words: Validity, reliability, metamemory, adult population.
\end{abstract}

\section{Introducción}

La valoración subjetiva de diversas dimensiones de la memoria proporciona información distinta al rendimiento objetivo de la misma y tiene importancia no sólo en contextos clínicos, sino también en investigación. Asimismo, contar con instrumentos válidos y confiables para evaluar diversos constructos de interés clínico y de investigación es de gran importancia para el desarrollo de las diversas disciplinas donde dichas mediciones tienen interés. La evaluación de aspectos del funcionamiento de la memoria ha tenido particular interés en población adulta y adultos

Nota autor: Este trabajo forma parte del proyecto Fondecyt 1090564, “Comprensión y producción de oraciones en adultos mayores. Estudio experimental en el marco del modelo procesamiento autorregulado del lenguaje", que desarrolla el grupo de investigación en Psicolingüística de la Universidad de Concepción. Correspondencia: Dr. Bernardo Riffo, Departamento de Español, Universidad de Concepción, Casilla 160-C, Concepción. E-mail: bernardo@udec.cl 
mayores (Mattos et al., 2003), existiendo actualmente diversos instrumentos como cuestionarios, escalas y aquellos que emplean tareas de recuerdo. En este sentido, gran parte de la investigación en metacognición y envejecimiento se ha centrado en el estudio de la metamemoria (Hertzog, 2002), entendida, de modo general, como el conocimiento y creencias que las personas tienen sobre el funcionamiento, evolución, limitaciones y capacidades de su propia memoria. La perspectiva de la evaluación de la metamemoria no niega la existencia de cambios en la memoria atribuibles a la edad, pero supone que dichas modificaciones en su funcionamiento se deben en parte a factores distintos al mero cambio ontogénico (Pérez, Peregrina, Justicia y Godoy, 1995).

Si bien existe un sesgo en la autoevaluación de la propia percepción del funcionamiento y confianza en la memoria, esta tiene una relación más cercana con los contextos cotidianos donde los fallos de memoria suelen ocurrir (Pérez et al., 1995), con lo que se obtiene una medida menos artificial que las de laboratorio. Esto, a su vez, permite orientar mejor el desarrollo de programas de intervención específicos (Troyer y Rich, 2002). Por otra parte, una desventaja relativa planteada por algunos autores es que los cuestionarios de creencias de memoria tendrían una validez predictiva limitada y una baja relación con el desempeño (Hertzog, 2002). Mattos et al. (2003) han señalado que los cuestionarios que preguntan directamente sobre problemas de memoria, al menos en población adulta sana, podrían entregar información más significativa que los cuestionarios de auto-reportes; sin embargo, debido a las limitaciones de sus datos, el problema requeriría más estudios específicos.

Entre los instrumentos empleados para estudiar la autopercepción del funcionamiento de la memoria destaca el Cuestionario de Metamemoria de Adultos (The Metamemory in Adulthood Questionnaire [MIA], Dixon, Hultsch y Hertzog, 1988) que contiene 108 ítems, distribuidos en siete subescalas, las que evalúan el conocimiento que las personas tienen del funcionamiento de su memoria, frecuencia de uso de estrategias para recordar, la capacidad de almacenamiento de la memoria, la percepción de su estabilidad en el tiempo, la ansiedad para recordar información y el locus de control en habilidad de memoria. La otra medida ampliamente utilizada es la que se obtiene con el Memory Functioning Questionnaire [MFQ] (Gilewski, Zelinski y Schaie, 1990). Estas pruebas se han complementado con cuestionarios y escalas que evalúan memoria cotidiana, es decir, las funciones de la memoria en situaciones ecológicas e incluyen además la percepción de los errores que se cometen y, en algunos casos, las quejas del sujeto respecto del funcionamiento de sus capacidades mnemónicas (Pérez et al., 1995; Troyer y Rich, 2002). Una de las limitaciones de dichas mediciones es que se desarrollaron con fines de investigación y no consideran necesariamente los aspectos del funcionamiento de la memoria que podrían ser relevantes para el contexto clínico y de evaluación. De igual forma, incluyen ítems que no necesariamente son aplicables a algunos individuos, como hablar en público o leer novelas, a su vez, la extensión de las pruebas, también puede ser considerada como una limitación, especialmente para personas mayores o con algún deterioro cognitivo (Troyer y Rich, 2002).

En este contexto, el Cuestionario Multifactorial de Memoria (Multifactorial Memory Questionnaire [MMQ]) de Troyer y Rich (2002) se propone superar las limitaciones arriba mencionadas y construye, basándose en escalas existentes, una nueva escala multidimensional, breve, con relevancia clínica, de fácil administración y adecuada validez y confiabilidad. Esta nueva escala puede ser usada, además, en contextos de investigación evaluando tres dimensiones de memoria auto-reportada, las que incluyen: a) satisfacción global con la propia capacidad de memoria y aspectos de la memoria relacionado con los afectos (Satisfacción), b) percepción de la capacidad de memoria cotidiana (Capacidad), y c) uso de estrategias y ayudas en recuerdo de eventos cotidianos (Estrategia).

En el estudio original, se seleccionó una muestra de 115 adultos entre 40 y 91 años $(M=71.7$ años, $D . E=9.9)$ con una educación promedio de 13.8 años. Luego de un análisis de componentes principales con rotación varimax, y forzando la solución a tres componentes, se encontró que estos, en conjunto, explican el $40.4 \%$ de la varianza total. Las tres escalas mostraron una adecuada estabilidad temporal test-retest, con cuatro semanas de diferencia, presentándose correlaciones de .93 para la sub-escala de Satisfacción, .86 para Capacidad y .88 para Estrategia. Se evaluó la confiabilidad de consistencia interna mediante el coeficiente Alfa de Cronbach (alfa de .95 para la sub-escala de Satisfacción, 93 para Capacidad y .83 para Estrategia).

El objetivo del presente estudio instrumental es examinar las propiedades psicométricas del MMQ en población adulta chilena que no presenta problemas de salud. Específicamente, nos proponemos evaluar confiabilidad y validez de las tres sub-escalas del instrumento. De particular interés para el equipo de investigación es la utilidad del cuestionario como medida para ser considerada, junto con otras, en estudios sobre las capacidades lingüísticas y cognitivas de adultos mayores. 


\section{Método}

\section{Participantes}

La muestra estuvo constituida por población adulta sana mayor de 20 años de la provincia de Concepción ${ }^{1}$. Los instrumentos fueron aplicados a un total de 823 personas, de las cuales $83(10.1 \%)$ fueron excluidos por presentar sintomatología depresiva, que, sin llegar a constituir un trastorno, sería indicio de depresión leve según puntaje de corte sugerido en versión original y adaptación chilena del Beck Depression Inventory Second Edition [BDI-II], (Beck, Steer y Brown, 1996; Melipillán, Cova, Rincón y Valdivia, 2008). Con esto, la muestra quedó finalmente compuesta por 740 personas de ambos sexos, $369(49.9 \%)$ mujeres y 371 (50.1\%) hombres. El rango de edad fue de 20 a 81 años, con un promedio de 38.3 años $(D . E .=14.3)$. Para algunos análisis se ha dividido la muestra en tres rangos de edad: 377 (50.9\%) personas corresponden a adultos jóvenes (20-35 años), 296 (40\%) adulto medio (36-60 años), y 67 (9.1\%) a adulto mayor (61 a 81 años). En relación con el nivel educacional, el $42 \%$ de los participantes tiene estudios superiores completos, mientras que un $23.6 \%$ corresponde a personas con estudios superiores incompletos, el $34.4 \%$ restante tiene enseñanza media completa. Respecto del nivel socioeconómico, $59.5 \%$ de los participantes se autocalificó como nivel medio y $35.8 \%$ medio alto, siendo marginales los niveles bajo y alto.

\section{Instrumentos}

\section{Cuestionario multifactorial de memoria}

Como se señaló antes, la versión original de The Multifactorial Memory Questionnaire [MMQ], objeto de estudio en la presente investigación, tiene un total de 57 ítems de auto-reporte tipo escala likert organizados en tres sub-escalas (Satisfacción, Capacidad y Estrategia) que evalúan distintos aspectos referidos a metamemoria, según se detalla a continuación.

MMQ-Satisfacción (ES): Contiene 18 ítems referidos a una serie de aspectos relacionados con emociones y percepciones del grado de satisfacción que el individuo tiene sobre su capacidad de memoria actual. Incluye emociones positivas como confianza y satisfacción por la memoria, emociones negativas como irritabilidad o vergüenza y evaluación subjetiva

La recolección de datos para este estudio se realizó entre diciembre de 2009 y enero de 2010, esto es, antes del terremoto del 27 de febrero del mismo año. de la habilidad como las creencias de tener problemas serios de memoria. Los ítems se responden en una escala de cinco puntos:1) muy de acuerdo, 2) de acuerdo, 3) indeciso, 4) en desacuerdo y 5) muy en desacuerdo, según el grado de congruencia con el ítem en relación con cómo las personas encuestadas se han sentido durante las dos últimas semanas. Se puntúa entre 0 y 4 puntos, de menor a mayor grado de coincidencia con el ítem. Un puntaje alto refleja mayor grado de satisfacción con el funcionamiento de la memoria. Los ítems $2,4,5,7,8,10,11,14,15,16$ y 18 se califican de forma inversa. Luego de un análisis estadístico inicial se decidió eliminar el ítem 9 de la escala que decía "cuando tengo problemas para recordar algo, no me exijo mucho", el que tuvo una baja correlación ítem-test total, bajo acuerdo entre jueces y no se ajustaba adecuadamente a la dimensión donde originalmente se había incluido. De esta forma, la sub-escala Satisfacción quedó conformada por 17 ítems.

$M M Q$-Capacidad (EC): Contiene un total de 20 preguntas que evalúan la autopercepción que el sujeto tiene del funcionamiento de su memoria en tareas cotidianas, como recordar un nombre o un número telefónico. Esta sub-escala se ha basado en cuestionarios anteriores, como MIA (Dixon et al., 1988) y el MFQ (Gilewski, Zelinski y Schaie, 1990). Las respuestas reflejan qué tan frecuente han sido algunas fallas de memoria las últimas dos semanas en una escala de frecuencia de cinco niveles. Un puntaje alto es indicativo de una mejor habilidad de memoria subjetiva, mientras que a menor puntaje es indicativo de una mayor falla de memoria cotidiana.

$M M Q$-Estrategia(EE): Corresponde a la tercera subescala, contiene 19 preguntas que describen diferentes ayudas y estrategias de memoria que las personas utilizan para recordar alguna información, referidas a tareas de memoria cotidiana (everyday memory tasks), tales como escribir una cita en un calendario o redactar una lista de deberes por hacer. Algunos de estos ítems están basados también en entrevistas y cuestionarios previos, como el MIA (Dixon et al., 1998). Las respuestas indican la frecuencia con que cada una de esas estrategias fue usada las últimas dos semanas, pudiendo responderse en una escala de nunca a siempre. Las respuestas son puntuadas de 0 a 4 puntos, donde un puntaje mayor es indicativo de una más alta frecuencia de uso de esa ayuda o estrategia.

\section{Cuestionario de funcionamiento de la memoria}

El Cuestionario de Funcionamiento de la Memoria [CFM] empleado en esta investigación, corresponde a una adaptación breve del MFQ, elaborado por Gilewski y sus colaboradores (Gilewski et al., 1990), que ha sido validada para población 
española adulta por Alarcón y Fernández (2008). Incluye 31 ítems de la escala original distribuidos en 15 ítems de frecuencia de olvidos cotidianos, 4 ítems de frecuencia de olvido durante la lectura, 4 ítems de recuerdos de eventos pasados y 8 ítems de uso de técnicas mnemotécnicas. Los reactivos se presentan en formato Likert de cinco niveles de respuesta distribuida de nunca a siempre. Debido a que no se cuenta con evidencia de validación del instrumento en población chilena, se calcularon indicadores de validez y confiabilidad, mostrando una estructura factorial de cuatro factores que explican el $50 \%$ de la varianza, similar a la presentada en población española y una alta consistencia interna (alfa de Cronbach de .79 para la escala total).

\section{Escala de satisfacción vital}

La escala de satisfacción vital (The Satisfaction with Life Scale [SWLS], Diener, Emmos, Larsen y Griffin, 1985), es una escala likert de siete niveles que evalúa el grado de acuerdo o descuerdo con cinco afirmaciones referidas a qué tan satisfecha se encuentra la persona con su vida, entendida como el componente subjetivo del bienestar. A mayor puntaje, más alta satisfacción. Los autores reportan, respecto de la versión original, una alta confiabilidad de consistencia interna $(\alpha=.87)$, siendo una escala unifactorial que explicaría el $66 \%$ de la varianza total (Diener et al., 1985), propiedades psicométricas que han sido confirmadas por otros estudios (Atienza, Pons, Belaguer, y Merita, 2000; Castro-Solano, 2000; Díaz-Morales, 2001; Neto, 1992). En este estudio, se modificó la escala reduciendo los niveles de respuesta de siete a cinco, sólo para hacerla consistente con los demás instrumentos incluidos y facilitar su completación, la modificación la justificamos bajo el argumento planteado por Lozano, García-Cueto y Muñiz (2008), quienes encontraron que el efecto en la confiabilidad de consistencia interna y validez factorial en escalas Likert con distintos niveles de respuesta no varía considerablemente entre 5 y 7 niveles. Un alto puntaje en esta escala es signo de una alta satisfacción vital, la que, según diversos estudios, está en directa relación con calidad de vida relacionada con la salud y su dimensión psicológica, más que física (Cabañero et al., 2004), así como con determinados estilos de personalidad (Díaz y Sánchez-López, 2001). Por el contrario, valores elevados correlacionan negativamente con depresión y medidas de afectos negativos (Chico y Ferrando, 2008).

\section{Escala de Depresión de Beck BDI-II}

Con el objeto de descartar a sujetos que pudieran presentar síntomas depresivos, se incluyó la adaptación española del inventario de depresión de Beck, BDI-II (Beck et al., 1996; Sanz, Navarro y Vázquez, 2003), un instrumento de 21 ítems que ha sido diseñado para evaluar la gravedad de sintomatología depresiva en población adolescente y adulta. En población chilena se han evaluado las propiedades psicométricas del instrumento en adolescentes, donde ha mostrado capacidad para discriminar entre población clínica y no clínica (Melipillán et al., 2008). En cada ítem de la escala, el sujeto debe elegir la frase que mejor describe su estado las últimas dos semanas, entre cuatro alternativas de menor a mayor gravedad, las que son puntuadas de $0 \mathrm{a}$ 4. La escala tiene un rango entre 0 y 63 puntos. Tal cual lo plantean los autores, se estableció como puntaje de corte 19 puntos, el que corresponde a depresión leve según criterios fijados por Beck en la versión original. Sobre 20 puntos se presentaría sintomatología clínica moderada y sobre 28, severa (Beck et al., 1996). Como se dijo antes, en este estudio se descartó a los participantes que obtuvieron 20 puntos o más, es decir, a 83 personas (10.1\%) de la muestra originalmente seleccionada, porcentaje que es levemente superior a la presencia de dicho trastorno en la región (Vicente, Khon, Rioseco, Saldivia, Navarrete et al., 2006; Vicente, Khon, Rioseco, Saldivia, Levav et al., 2006). En cuanto a la confiabilidad, el BDI-II obtuvo en este estudio un coeficiente alfa de Cronbach de .90 .

Para la presente investigación, se ha descartado específicamente mediante este test los cuadros depresivos, por ser uno de los más prevalentes en la población y tener mayor relación con las funciones de memoria. Otros trastornos psiquiátricos han sido descartados solo al no reportar los participantes problemas de salud.

\section{Cuestionario de variables sociodemográficas}

Finalmente, se incluyó en la investigación un cuestionario elaborado ad hoc, el que incluye variables sociodemográficas como sexo, edad, nivel educacional, estado civil, nivel socioeconómico, nivel de satisfacción con la vida y una autoevaluación de su estado general de salud, las que permitieron segmentar la muestra y establecer relaciones con las diversas sub-escalas de memoria. 


\section{Procedimiento}

El conjunto de instrumentos fue organizado para que estos puedan ser autoaplicados en un formato único en el orden que se indica: Cuestionario sociodemográfico, MMQ, SWLS, CFM y BDI-II. El tiempo promedio de aplicación fue de 20 minutos, y en el caso de personas mayores podían ser asistidas para marcar sus respuestas, o bien aplicar el instrumento como entrevista.

La validez de contenido del MMQ fue establecida mediante juicio de expertos, previa adaptación idiomática y traducción inversa (back-translation) a la que fue sometido el instrumento, al mismo tiempo que se realizó una aplicación piloto de acuerdo a recomendaciones generales propias de este tipo de estudios (Arribas, 2006; Balluerka, Gorostiaga, Alonso-Arbiol y Haranburu, 2007; Carretero-Dios y Pérez, 2007). La validez factorial se estableció mediante Análisis Factorial Exploratorio (AFE). La evidencia de validez concurrente de las escalas se determinó con la correlación producto-momento de Pearson con los puntajes entre cada sub-escala con su equivalente en el CFM. La evaluación de confiabilidad de cada una de las escalas se llevó a cabo sobre la base del análisis de consistencia interna a través de la estimación del coeficiente alfa de Cronbach. Para el análisis estadístico de los datos se utilizó el software SPSS 18 (Chicago, IL, IBM) y el programa FACTOR 7.00 (Lorenzo-Seva y Ferrando, 2006).

\section{Resultados}

\section{Confiabilidad}

El coeficiente Alfa de Cronbach basado en elementos tipificados para cada una de las subescalas fue de .88 para ES, .91 para EC y .89 para EE, siendo de .82 para la escala total. En todos los casos los índices de correlación ítem-test total fueron altos, excepto el ítem 9 de la ES que fue eliminado. Los valores alfa corregidos obtenidos fueron aceptables y similares entre ellos; así, la eliminación de cualquiera de los ítems restantes no produce un aumento significativo en la consistencia interna de cada una de las subescalas, por lo que no fueron eliminados otros ítems.

\section{Validez de contenido}

Se evaluó la validez de contenido de los distintos ítems incluidos en cada una de las escalas del MMQ con consulta a 15 jueces expertos, procedimiento similar a la validación original, donde se empleó como criterio un 70\% de acuerdo de 12 expertos en memoria (Troyer y Rich, 2002). Para el presente estudio, 14 de los 15 jueces eran psicólogos, de los cuales 12 tenían estudios de postgrado; el otro juez correspondió a un médico con formación en neuropsicología. Debido a que los jueces no eran expertos en memoria específicamente y, a pesar de que los ítems en el instrumento original ya habían sido clasificados, se decidió aumentar el criterio a un $85 \%$ de acuerdo, con el propósito de obtener mayor certeza sobre los resultados. De esta forma, 20 de los 57 ítems obtuvieron un 100\% de acuerdo, los restantes ítems fueron clasificados en la categoría correspondiente al menos con un $85 \%$ de acuerdo entre jueces, exceptuando el ítem 9 de la ES, el que fue calificado, al menos una vez, en las tres sub-escalas.

Para el proceso de recolección de los datos, se les proporcionó a los jueces un formato con una definición general para cada sub-escala y los 57 ítems fueron distribuidos al azar, donde cada juez debía clasificar cada ítem en alguna sub-escala. Se les solicitaba, además, sugerencias para la corrección de los ítems, siendo la observación más recurrente el que algunos ítems de capacidad incluían conceptos que aludían a la emoción, y podrían ser considerados en la sub-escala de satisfacción. Esto permitió, a su vez, evaluar la relación entre los contenidos y el constructo, y valorar los factores internos o externos que podrían añadir varianza no deseada (Elousa, 2003).

\section{Validez factorial}

Sobre la base de las respuesta relativas a los 56 ítems conservados, correspondientes a las tres sub-escalas, se efectuó un Análisis de Componentes Principales (ACP), que, aunque presenta algunas limitaciones en comparación con el Análisis Factorial propiamente tal, reporta una solución similar a la obtenida, por ejemplo, a través del método del Análisis Factorial de Ejes Principales, esto debido al tamaño de la muestra (Ferrando, 1996; Velicer y Jackson, 1990).

La medida de adecuación muestral de Kaiser-Meyer-Olkin (KMO) fue de 0.93 , la prueba de esfericidad de Bartlett señala una alta dependencia entre los ítems $\left[\chi_{(1485)}^{2}=15641.2, p<.001\right]$, siendo el determinante de la matriz muy cercano a 0 , lo que nos lleva a concluir que el ACP resulta, a priori, pertinente. Se usó como método de extracción el ACP y como procedimiento para determinar el número de dimensiones el Análisis Paralelo con re-muestreo a partir de los marginales (Parallel Analysis Using Marginally Bootstrapped Samples, PA-MBS) propuesto por Lattin, Carroll y Green (2003). Se forzó la solución a tres 
componentes, con rotación Varimax, cargando la totalidad de los ítems de cada subescala a uno de los tres componentes. Los resultados arrojan cargas factoriales superiores a 0.30 en la gran mayoría de los ítems de las sub-escalas, salvo en los ítems 3 y 12 de la ES que fue de 0.30 . Pese a ello, se decidió mantener dichos ítems en la escala. El análisis mostró que los tres componentes principales extraídos explican el $23.7 \%, 7.8 \%$ y $4.8 \%$ de la varianza total respectivamente $\mathrm{y}$, en conjunto, los factores dan cuenta del $36.3 \%$ de la varianza total. Cada uno de los ítems presentó una carga factorial claramente asociada a un factor, que fluctúa entre 0.30 y 0.68 para la ES, $0.37-0.66$ para EE y 0.46-0.66 para la EC. La carga factorial y comunalidad para cada uno de los ítems se presentan en la tabla 1.

\section{Validez convergente}

Con el objeto de obtener datos para determinar la validez convergente de las tres sub-escalas del MMQ, se procedió

Tabla 1. Cargas factoriales y comunalidad final de los items por sub escalas, autovalor y porcentaje de varianza explicada para cada componente del Cuestionario de Memoria Multidimensional.

\begin{tabular}{|c|c|c|c|c|c|c|}
\hline \multirow[b]{2}{*}{ ítem } & \multicolumn{2}{|c|}{ Estrategia } & \multicolumn{2}{|c|}{ Capacidad } & \multicolumn{2}{|c|}{ Satisfacción } \\
\hline & Carga & $h^{2}$ & Carga & $h^{2}$ & Carga & $h^{2}$ \\
\hline 1 & 0.37 & 0.17 & 0.56 & 0.32 & 0.59 & 0.41 \\
\hline 2 & 0.43 & 0.40 & 0.50 & 0.29 & 0.65 & 0.46 \\
\hline 3 & 0.52 & 0.29 & 0.46 & 0.35 & 0.30 & 0.05 \\
\hline 4 & 0.56 & 0.32 & 0.53 & 0.36 & 0.60 & 0.38 \\
\hline 5 & 0.58 & 0.35 & 0.57 & 0.38 & 0.61 & 0.41 \\
\hline 6 & 0.47 & 0.25 & 0.51 & 0.32 & 0.56 & 0.38 \\
\hline 7 & 0.59 & 0.36 & 0.57 & 0.41 & 0.68 & 0.49 \\
\hline 8 & 0.61 & 0.41 & 0.66 & 0.45 & 0.62 & 0.40 \\
\hline 9 & 0.49 & 0.27 & 0.52 & 0.39 & 0.66 & 0.47 \\
\hline 10 & 0.61 & 0.41 & 0.49 & 0.38 & 0.63 & 0.51 \\
\hline 11 & 0.61 & 0.39 & 0.51 & 0.29 & 0.58 & 0.37 \\
\hline 12 & 0.57 & 0.42 & 0.55 & 0.35 & 0.30 & 0.06 \\
\hline 13 & 0.66 & 0.47 & 0.56 & 0.34 & 0.44 & 0.23 \\
\hline 14 & 0.59 & 0.38 & 0.59 & 0.46 & 0.68 & 0.49 \\
\hline 15 & 0.64 & 0.43 & 0.52 & 0.32 & 0.37 & 0.19 \\
\hline 16 & 0.53 & 0.28 & 0.50 & 0.30 & 0.59 & 0.39 \\
\hline 17 & 0.62 & 0.41 & 0.49 & 0.26 & 0.66 & 0.48 \\
\hline 18 & 0.61 & 0.42 & 0.62 & 0.46 & -- & -- \\
\hline 19 & 0.49 & 0.39 & 0.61 & 0.43 & -- & -- \\
\hline 20 & -- & -- & 0.62 & 0.46 & -- & -- \\
\hline Autovalor & \multicolumn{2}{|c|}{13.24} & \multicolumn{2}{|c|}{4.38} & \multicolumn{2}{|c|}{2.67} \\
\hline \% V.E. & \multicolumn{2}{|c|}{23.65} & \multicolumn{2}{|c|}{7.83} & \multicolumn{2}{|c|}{4.77} \\
\hline
\end{tabular}

Nota. $\%$ V.E. $=$ Porcentaje de varianza explicada para cada factor. a aplicar, en conjunto con este cuestionario, el CFM, en su versión española, como se señala en la sección anterior (Alarcón y Fernández, 2008). Se estimó la correlación productomomento de Pearson para los puntajes de cada una de las sub-escalas de ambos instrumentos, los resultados pueden observarse en la tabla 2. En términos generales, y como se esperaba, existe una correlación directa entre las sub-escalas que evalúan estrategias para recordar información $(r=.71$, $p<.001)$; la EC del MMQ es, a su vez, equivalente a la de memoria cotidiana del CFM, con una correlación inversa $(r=-.81, p<.001)$, lo que se explica por el hecho de que una mide los errores de memoria en situaciones cotidianas, y la otra la frecuencia con que no se comenten dichos errores. Sólo las EC y EE del MMQ son equivalentes con las subescalas de memoria cotidiana y estrategias nemotécnicas del CFM. La ES no tiene equivalente funcional, por lo que no es posible hacer alguna comparación.

\section{Análisis descriptivo de la escala y relación entre variables demográficas y metamemoria}

La tabla 3 muestra los valores de las medias, desviaciones estándar de las puntuaciones y percentiles de cada una de las subescalas del MMQ para la totalidad de la muestra. La escala se aplica a población adulta procurando abarcar todos los rangos etarios, los que se han dividido en rangos para facilitar la interpretación de los datos correspondientes a cada uno de ellos.

$\mathrm{Al}$ estimar diferencia de sexo a través de la prueba $t$ de Student para muestras independientes, con un nivel de confianza de un $95 \%$, es posible establecer que no existen diferencias estadísticamente significativas entre hombres y mujeres en las respuestas de las ES $\left[t_{(709.147)}=1.884, p<\right.$ $.060, d$ de Cohen $=.14]$ y EC $\left[t_{(722.129)}=1.610, p<.108, d\right.$ de Cohen $=.12]$; sin embargo, sí se presentan diferencias estadísticamente significativas en la sub-escala de uso de estrategias, donde son las mujeres quienes con mayor frecuencia emplean diversas estrategias para recordar información $\left[t_{(738)}=3.996, p<.003, d\right.$ de Cohen $\left.=.29\right]$.

Al correlacionar la edad con los puntajes totales de las sub-escalas, se observa una correlación inversa con la $\mathrm{ES}(r=-.20, p<.001)$ y la EC $(r=-.20, p<.001)$, existiendo una muy baja correlación con la EE $(r=.09$, $p<.02)$. El análisis según rangos, por su parte, muestra diferencias en las ES y EC, no así en EE. De esta forma, son las personas más jóvenes quienes presentan mejores niveles de satisfacción con su capacidad de memoria y cometen menos errores. En ambos casos, las diferencias se producían entre el grupo más joven y los 
Tabla 2. Correlación producto-momento de Pearson entre las subescalas del Multifactorial Memory Questionnaire (MMQ) y el Cuestionario de funcionamiento de la memoria (CFM).

\begin{tabular}{lccccccc}
\hline Subescalas & 1 & 2 & 3 & 4 & 5 & 6 & 7 \\
\hline 1. Satisfacción & -- & & & & & & \\
2. Capacidad & .57 & -- & & & & & \\
3. Estrategia & -.34 & -.52 & -- & & & & \\
4. Memoria cotidiana & -.50 & -.81 & .50 & -- & & & \\
5. Recuerdo de textos & -.41 & -.45 & .27 & .49 & -- & & \\
6. Recuerdo del pasado & .25 & -.28 & -.16 & -.28 & -.20 & -- & - \\
7. Estrategias nemotécnicas & -.23 & -.34 & .71 & -.33 & .19 & $-.08^{*}$ & -- \\
\hline
\end{tabular}

Nota. Basado en 740 participantes. 1,2,3 = Subescalas MMQ; 4,5,6,7 = Subescalas CFM. Todas las correlaciones son significativas al nivel de .001 (bilateral), exceptuando la marcada con* que lo es al .05 (bilateral).

Tabla 3. Resultados descriptivos generales para cada unas de las subescalas del MMQ según rangos de edad.

\begin{tabular}{|c|c|c|c|c|c|c|c|c|c|}
\hline & \multicolumn{3}{|c|}{$\begin{array}{l}\text { Adulto joven } \\
(\mathrm{n}=377)\end{array}$} & \multicolumn{3}{|c|}{$\begin{array}{l}\text { Adulto medio } \\
(\mathrm{n}=296)\end{array}$} & \multicolumn{3}{|c|}{$\begin{array}{l}\text { Adulto mayor } \\
(\mathrm{n}=67)\end{array}$} \\
\hline & ES & $\mathrm{EC}$ & $\mathrm{EE}$ & ES & $\mathrm{EC}$ & $\mathrm{EE}$ & ES & $\mathrm{EC}$ & $\mathrm{EE}$ \\
\hline Media & 50.52 & 59.42 & 21.22 & 46.81 & 55.94 & 21.51 & 45.96 & 55.33 & 23.39 \\
\hline D.E. & 9.74 & 9.15 & 11.15 & 10.96 & 10.51 & 12.28 & 11.41 & 12.43 & 13.18 \\
\hline Rango & 65 & 57 & 67 & 62 & 61 & 57 & 61 & 57 & 57 \\
\hline \multicolumn{10}{|c|}{ Percentiles } \\
\hline 1 & 20.8 & 31.9 & 0 & 17.9 & 30.7 & 0 & 7.0 & 23.0 & 1.0 \\
\hline 5 & 30.0 & 43.0 & 3.9 & 26.9 & 38.0 & 4 & 25.8 & 33.4 & 3.8 \\
\hline 25 & 45.0 & 54.0 & 13.0 & 40.0 & 49.0 & 12.0 & 38.0 & 48.0 & 14.0 \\
\hline 50 & 52.0 & 59.0 & 20.0 & 48.0 & 57.0 & 20.0 & 46.0 & 56.0 & 22.0 \\
\hline 75 & 57.5 & 66.0 & 28.0 & 54.0 & 63.0 & 30.0 & 53.0 & 64.0 & 30.0 \\
\hline 95 & 64.0 & 74.0 & 43.0 & 65.0 & 72.0 & 44.0 & 65.6 & 75.8 & 51.6 \\
\hline 99 & 68.0 & 78.2 & 51.0 & 67.0 & 79.0 & 55.0 & 68.0 & 80.0 & 58.0 \\
\hline
\end{tabular}

Nota . Adulto joven $=20-35$ años, adulto medio $=36-60$ años, adulto mayor $=61-81$ años. ES $=$ Subescala Satisfacción; EC $=$ Subescala Capacidad; E = Subescala Estrategia.

grupos mayores, no habiendo diferencia entre el grupo de mediana edad con los mayores de 60 años estimado a través de la prueba post hoc HSD Tukey. En el uso de estrategias nemotécnicas, si bien a mayor edad hay una tendencia a usar más frecuentemente dichas estrategias, no se observa una diferencia estadísticamente significativa entre ninguno de los tres grupos. Los resultados pueden observarse en tabla 4.

En un análisis según nivel educacional, se aprecian diferencias estadísticamente significativas entre los diversos niveles de estudio $(p<.001)$. En la medida que el nivel educacional aumenta, existe una mayor satisfacción con la

Tabla 4. Análisis de varianza para cada subescala del MMQ y variables relacionadas.

\begin{tabular}{|c|c|c|c|c|c|c|c|c|c|}
\hline \multirow[b]{2}{*}{ Variables } & \multicolumn{3}{|c|}{ Satisfacción } & \multicolumn{3}{|c|}{ Capacidad } & \multicolumn{3}{|c|}{ Estrategia } \\
\hline & $F$ & $p$ & $\eta^{2}$ & $F$ & $p$ & $\eta^{2}$ & $F$ & $p$ & $\eta^{2}$ \\
\hline Edad* & 12.996 & .001 & .034 & 11.938 & .001 & .031 & 0.960 & .383 & .003 \\
\hline Nivel educacional & 5.683 & .001 & .030 & 4.118 & .001 & .022 & 3.497 & .008 & .019 \\
\hline Nivel socioeconómico & 5.361 & .001 & .021 & 2.067 & .103 & .008 & .440 & .724 & .002 \\
\hline Salud subjetiva & 11.887 & .001 & .061 & 12.659 & .001 & .064 & 3.147 & .014 & .017 \\
\hline Satisfacción vital & 5.219 & .001 & .028 & 2.651 & .032 & .014 & 0.290 & .884 & .002 \\
\hline
\end{tabular}

Nota. * Edad en rangos: 20-35 años; 36-60 años, 61-81 años. 
capacidad de memoria, disminuyen los errores de desempeño y hay un menor uso de estrategias mnemónicas.

En relación con el nivel socioeconómico, no se presentaron diferencias estadísticamente significativas en la sub-escala de capacidad de memoria ni de uso de estrategias; en cambio, sí se hallaron diferencias en relación con la dimensión satisfacción, donde a mayor nivel socioeconómico existe un mayor puntaje en dicha dimensión $(p<.001)$.

Finalmente, a partir del nivel subjetivo de salud reportado por los participantes en una escala de cinco niveles que permitía calificar su estado de salud de malo a excelente, es posible establecer que existen diferencias estadísticamente significativas en las tres sub-escalas; mientras más alto es el estatus de salud reportado, mayor es el nivel de satisfacción. Lo mismo ocurre con la capacidad de memoria, donde se obtienen mayores puntajes a mayor estatus de salud y, por el contrario, disminuyen las estrategias para recordar información cuando se percibe una mejor salud. Esta diferencia se presenta incluso luego de haber sido eliminados los participantes que expresaron tener una mala salud y que coincidían con altos puntajes en el BDI-II que no habían sido excluidos de la muestra.

Respecto del grado de satisfacción vital medido con el SWLS, se encontraron diferencias estadísticamente significativas en dos de las tres sub-escalas: un mayor nivel de satisfacción vital concuerda con un más alto puntaje en la sub-escala Satisfacción y en Competencia. Esta diferencia no se presentó en la sub-escala Estrategia.

\section{Discusión}

La escala MMQ es un instrumento que ha demostrado una alta consistencia interna en sus tres sub-escalas, al mismo tiempo que presenta indicadores de validez satisfactorios. Los resultados de la presente investigación permiten afirmar que la prueba mantiene las propiedades psicométricas reportadas en el estudio original con población adulta norteamericana (Troyer y Rich, 2002), obteniéndose indicadores similares en población chilena de similar edad. Por otra parte, se ha extendido su aplicación a población adulta joven, lo que permite hacer una comparación de los procesos en diversos estadios de la adultez y facilitar así estudios con una población más amplia comparando el constructo medido a lo largo de la vida. Tales posibilidades traerán, además, no sólo beneficios en términos de su aplicación en investigación, sino también en su utilización en contextos clínicos y académicos.
De la evaluación de los ítems de cada sub-escala, sólo fue eliminado el ítem 9 de la ES ("cuando tengo problemas para recordar algo, no me exijo mucho"). Esta decisión se debió a diversas razones: se presentó una baja correlación ítem-test afectando la consistencia interna de la escala $(r$ $=-.099$ ). En la evaluación de jueces, este ítem fue el que presentó mayor discrepancia, clasificado aproximadamente la mitad de las veces en la ES y otras en EE o EC. Este ítem presentó además una baja carga factorial dentro del componente al que pertenecía (menor a 0.3 ). Se exploraron diversas soluciones mediante análisis de clúster jerárquicos; sin embargo, no fue posible asociarlo a ningún clúster de forma consistente, todo lo cual llevó a excluirlo, quedando finalmente conformada la ES por 17 ítems.

En cuanto a la estructura factorial, nuestros resultados son levemente diferentes a los presentados por Troyer y Rich (2002). Específicamente, encontramos que el porcentaje de varianza explicada para cada uno de los componentes es levemente inferior al reportado por los autores; sin embargo, resulta además relevante que la EE fue la que corresponde al componente 1 , mientras que en el estudio original corresponde a ES. Esta diferencia se debe, en parte, a elementos de carácter metodológico y posiblemente cultural. Entre los primeros, hay que considerar que la escala fue aplicada a un rango de edad más amplio que el estudio original, tomando a personas de 20 a 39 años, grupo etario que no había sido incluido en el la investigación de Troyer y Rich (2002), donde solo se incluyó a adultos mayores de 40 años. En segundo término, está el efecto asociado al análisis factorial exploratorio, donde los autores norteamericanos hacen uno más convencional, mientras que en nuestro caso se utiliza una variante del análisis paralelo, recomendado por las ventajas que tendría sobre el más convencional (Lorenzo-Seva y Ferrando, 2006).

Entre las posibles limitaciones del presente estudio debemos señalar dos. En primer lugar, la población adulta mayor de 60 años está sub-representada en relación con los otros dos niveles, lo que puede ser mejorado en estudios futuros. En segundo lugar, si bien el instrumento surge como un esfuerzo por unificar diversas medidas de metamemoria en una escala más breve, consideramos que es posible homogeneizar aún más el MMQ utilizando una sola escala likert con tres dimensiones, lo que obligaría a convertir la EC y EE de preguntas a afirmaciones y modificar las opciones de respuesta a una escala de nivel de acuerdo; además, se puede mantener el nivel de consistencia interna con una posible reducción de ítems, de momento hemos decidido mantener dos ítems con una carga factorial de 0.30 en la escala de ES por sustentarse teóricamente. 
Finalmente, podemos concluir que las diversas evidencias aportadas en este estudio inicial permiten afirmar que la escala MMQ es un instrumento válido y confiable que puede usarse en población chilena adulta, tanto con fines de evaluación clínica como de investigación.

\section{Referencias}

Alarcón, D., y Fernández, C. (2008). Validación de la versión abreviada en español del Cuestionario de Funcionamiento de la Memoria (CFM) en una población mayor de 55 años. Anales de Psicología, 24, 320-326.

Arribas, A. (2006). Adaptación transcultural de instrumentos. Guía para el proceso de validación de instrumentos tipo encuestas. Revista Cientifica de la $A M B B, 16,74-82$.

Atienza , F. L., Pons, D., Balaguer, I., y García-Merita, M. (2000). Propiedades psicométricas de la escala de Satisfacción con la vida en adolescentes. Psicothema, 12, 314-319.

Beck, A. T., Steer, R. A., y Brown, G. K. (1996). BDI-II. Beck Depression Inventory-second edition. Manual. San Antonio: The Psychological Corporation.

Balluerka, N., Gorostiaga, A., Alonso-Arbiol, I., y Haranburu, M. (2007). La adaptación de instrumentos de medida de una cultura a otras: una perspectiva práctica. Psicothema, 19, 124-133.

Cabañero, M., Martínez, M., Cabrero, J., Ortis, M. Reig, A., y Tosal, B. (2004). Fiabilidad y validez de la escala de satisfacción con la vida de Diener en una muestra de mujeres embarazadas y Puérperas. Psicothe$m a, 16,448-455$.

Carretero-Dios, H., y Pérez, C. (2007). Normas para el desarrollo y revisión de estudios instrumentales: consideraciones sobre la selección de tests en la investigación psicológica. International Journal of Clinical and Health Psychology, 7, 863-882.

Castro-Solano, A. (2000). Estilos de personalidad, objetivos de vida y satisfacción vital. Un estudio comparativo con adolescentes argentinos. (Tesis Doctoral). Universidad Complutense de Madrid. España.

Chico, E., y Ferrando, P. (2008). Variables cognitivas y afectivas como predictoras de satisfacción en la vida. Psicothema, 20, 408-412.

Díaz-Morales, J. F. (2001). Diferencias intergrupales en el sistema de metas personales, satisfacción y rendimiento en adolescentes. (Tesis Doctoral). Universidad Complutense de Madrid. España.

Díaz, J. F., y Sánchez-López, M. P. (2001). Relevancia de los estilos de personalidad y las metas personales en la predicción de la satisfacción vital. Anales de psicología, 17, 151-158.

Diener, E., Emmons, R., Larsen, R., y Griffin, S. (1985). The Satisfaction With Life Scale. Journal of Personality Assessment, 49, 71-75.

Dixon, R. A., Hultsch, D. F., y Hertzog, C. (1988). The Metamemory in Adulthood (MIA) Questionnaire. Psychopharmacology Bulletin, 24 671-688.

Elousa, P. (2003). Sobre la validez de los test. Psicothema, 15, 315-321.

Ferrando, P. J. (1996). Evaluación de la unidimensionalidad de los ítems mediante análisis factorial. Psicothema, 8, 397-410.

Gilewski, M. J., Zelinski, E., y Schaie, K. W. (1990). The metamemory Functioning Questionnaire for Assessment of memory complaints in adulthood and old age. Psychology and Aging, 5, 482-490.

Hertzog, C. (2002). Metacognition in older adults: implications for application. In T. Perfect y B. Schwartz (Eds.), Applied metacognition (pp.169-196). United Kingdom: Cambridge University Press.

Lattin, J., Carroll, D. J., y Green, P. E. (2003). Analyzing multivariate data. California, USA: Duxbury Press.

Lorenzo-Seva, U., y Ferrando, P. J. (2006). FACTOR: A computer program to fit the exploratory factor analysis model. Behavioral Research Methods, Instruments and Computers, 38, 88-91.
Lozano, L. M., García-Cueto, E., y Muñiz, J. (2008). Effect of the number of response categories on the reliability and validity of rating scales. Methodology, 4, 73-79.

Mattos, P., Lino, V., Rizo, L., Alfano, A., Araújo, C., y Raggio, R. (2003). Memory complaints and test performance in healthy elderly persons. Arquivos de Neuropsiquiatría, 61, 920-924

Melipillán, R., Cova, F., Rincón, P., y Valdivia, M. (2008). Propiedades psicométricas del inventario de Depresión de Beck-II en adolescentes chilenos. Terapia Psicológica, 26, 59-69.

Neto, F. (1992). The Satisfaction with life scale: Psychometrics properties in a Adolescent sample. Journal of Youth and Adolescence, 22, 125-134.

Pérez, M., Pelegrina, S., Justicia, F., y Godoy, J. (1995). Memoria cotidiana y Metamemoria en ancianos Institucionalizados. Anales de Psicología, 11, 47-62.

Sanz, J., Navarro, M. E., y Vázquez, C. (2003). Adaptación española del inventario para la depresión de Beck-II (BDI-II):1. Propiedades psicométricas en estudiantes universitarios. Análisis y Modificación de Conducta, 29, 239-288.

Troyer, A., y Rich, J. (2002). Psychometric properties of a new metamemory questionnaire for older adults. Journal of gerontology: psychological sciences, 57, 19-27.

Velicer, W. F., y Jackson, D. N. (1990). Component analysis versus common factor analysis: some issues in selecting an appropriate procedure. Multivariate Behavioral Research, 25, 1-29.

Vicente, B., Khon, R., Rioseco, P., Saldivia, S., Navarrete, G., Veloso, P., y Torres, S. (2006). Regional differences in psychiatric disorders in Chile. Social Psychiatry and Psychiatric Epidemioly, 41, 935-942. doi: 10.1007/s00127-006-0124-5.

Vicente, B., Khon, R., Rioseco, P., Saldivia, S., Levav, I., y Torres, S. (2006). Lifetime and 12-month prevalence of DSM-III-R disorders in the Chile Psychiatric prevalence study. The American Journal of Psychiatry, 163, 1362-1370.

\section{Anexos}

A continuación se presentan los ítems incluidos en la versión final del Cuestionario Multifactorial de Memoria (MMQ) para cada sub escala.

\section{MMQ-Satisfacción}

1. Generalmente me siento contento(a) con mi capacidad de memoria

2. Realmente tengo problemas graves con mi memoria.

3. Si algo es importante, probablemente lo recordaré.

4. Cuando olvido algo, temo que tal vez tenga algún problema grave de memoria, como el mal de Alzheimer.

5. Mi memoria es peor que la de la mayor parte de las personas de mi edad.

6. Confío en mi habilidad para recordar cosas.

7. No me siento contento(a) cuando pienso en mi capacidad de memoria.

8. Me preocupa que los demás se den cuenta de que mi memoria no es muy buena.

9. Estoy preocupado(a) por mi memoria. 
10. En realidad, mi memoria está decayendo últimamente.

11. Generalmente me siento satisfecho(a) con mi capacidad de memoria.

12. No me molesta cuando tengo problemas para recordar algo.

13. Me preocupa que pueda olvidar algo importante.

14. Mi capacidad de memoria me da vergüenza

15. Me enojo o irrito conmigo mismo(a) cuando olvido algo.

16. Mi memoria es buena para la edad que tengo.

17. Me preocupa mi capacidad de memoria.

\section{MMQ-Competencia}

1. ¿Con qué frecuencia olvida pagar a tiempo una cuenta?

2. ¿Con qué frecuencia deja en el lugar equivocado algo que usa a diario, como sus llaves o sus anteojos?

3. ¿Con qué frecuencia tiene problemas para recordar un número de teléfono que acaba de buscar?

4. ¿Con qué frecuencia no recuerda el nombre de alguien que acaba de conocer?

5. ¿Con qué frecuencia se le olvida llevar algo que tenía intención de llevar?

6. ¿Con qué frecuencia olvida una cita?

7. ¿Con qué frecuencia olvida algo que estaba a punto de hacer, como, por ejemplo, entrar a una habitación y olvidar lo que iba a hacer allí?

8. ¿Con qué frecuencia olvida hacer una diligencia?

9. ¿Con qué frecuencia tiene problemas para recordar una palabra específica que necesita?

10. ¿Con qué frecuencia tiene problemas para recordar un artículo de un diario o una revista que ha leído durante el mismo día?

11. ¿Con qué frecuencia se le olvida tomarse sus remedios?

12. ¿Con qué frecuencia no recuerda el nombre de alguien que conoce desde hace ya algún tiempo?

13. ¿Con qué frecuencia olvida dar un recado?

14. ¿Con qué frecuencia olvida algo que iba a decir en una conversación?

15. ¿Con qué frecuencia olvida un cumpleaños o un aniversario que solía recordar bien?

16. ¿Con qué frecuencia olvida un número de teléfono que usa a menudo?
17. ¿Con qué frecuencia vuelve a contar una historia o un chiste a la misma persona porque se le olvida que ya se lo había contado?

18. ¿Con qué frecuencia olvida dónde puso algo que guardó hace unos pocos días?

19. ¿Con qué frecuencia olvida algo que tenía la intención de comprar?

20. ¿Con qué frecuencia olvida los detalles de una conversación reciente?

\section{MMQ-Estrategia}

1. ¿Con qué frecuencia usa un reloj o una alarma para recordar el momento en que debe hacer algo?

2. ¿Con qué frecuencia le pide a alguien que le ayude a acordarse de algo o que le recuerde que debe hacer algo?

3. ¿Con qué frecuencia inventa una rima para algo que desea recordar?

4. ¿Con qué frecuencia crea una imagen visual para recordar algo, como por ejemplo, un nombre o un rostro?

5. ¿Con qué frecuencia anota en un calendario o agenda las citas o cosas que necesita hacer?

6. ¿Con qué frecuencia repasa el abecedario letra por letra para ver si le ayuda a recordar un nombre o una palabra?

7. ¿Con qué frecuencia organiza la información que desea recordar, por ejemplo, la lista del supermercado ordenada por tipos de alimentos?

8. ¿Con qué frecuencia dice algo en voz alta para recordarlo, como por ejemplo, un número de teléfono que acaba de buscar?

9. ¿Con qué frecuencia utiliza una rutina para recordar cosas importantes, como, por ejemplo revisar si tiene las llaves o la billetera al salir de casa?

10. ¿Con qué frecuencia hace listas, tal como una lista de compras o una lista de cosas por hacer?

11. ¿Con qué frecuencia se esfuerza en poner mucha atención en algo que desea recordar, por ejemplo, concentrándose mucho en los detalles?

12. ¿Con que frecuencia pone un objeto en un lugar visible para recordar que debe hacer algo, como poner el paraguas frente a la puerta para recordar que debe llevarlo?

13. ¿Con qué frecuencia se repite algo a sí mismo(a) a intervalos cada vez más prolongados con el fin de recordarlo?

14. ¿Con qué frecuencia crea una historia para enlazar información que desea recordar? 
15. ¿Con qué frecuencia anota en una libreta o agenda cosas que desea recordar?

16. ¿Con qué frecuencia crea un acrónimo con la primera letra de una lista de cosas que recordar, como por ejemplo, papas, azúcar, nueces (pan)?

17. ¿Con qué frecuencia se concentra mucho e intencionadamente en algo con el fin de recordarlo?
18. ¿Con qué frecuencia escribe una nota o un recordatorio para usted mismo (en un lugar distinto de un calendario o una libreta)?

19. ¿Con qué frecuencia vuelve sobre sus pasos con la intención de recordar algo, como por ejemplo, la ubicación de un objeto que no recuerda dónde lo dejó? 
